\title{
EXERCícIO DE INTENSIDADE MODERADA ASSOCIADO À DIETA CETOGÊNICA. UM MODELO DE ESTUDO PARA O METABOLISMO DE AMÔNIA?
}

\author{
Flavio Bachini'1,2 f.bachini@gmail.com \\ João Pedro Saar Werneck-de-Castro',3 joaopedrowerneck@pq.cnpq.br \\ Leonardo Cristiano Moretzsohn" ${ }^{1,4}$ leo.c.moretzsohn@bol.com.br \\ Adriana Bassini-Cameron' adriana.bassini@terra.com.br \\ Luiz-Claudio Cameron ${ }^{1,4,5}$ cameron@unirio.br
}

doi:10.3900/fpi.8.3.226.p

Bachini F, Werneck-de-Castro JPS, Moretzsohn LC, Bassini-Cameron A, Cameron L-C. Exercício de intensidade moderada associado à dieta cetogênica. Um modelo de estudo para o metabolismo de amônia? Fit Perf J. 2009 mai-jun;8(3):226-32.

\section{RESUMO}

Introdução: $O$ exercício tem sido utilizado como modelo para compreender a produção de amônia $\left(\mathrm{NH}_{3}+\mathrm{NH}_{4}{ }^{+}\right)$ um metabólito tóxico para o sistema nervoso central. A dieta cetogênica é caracterizada pela redução no consumo de carboidratos levando modificações metabólicas para manutenção da relação ATP/ADP, que incluem a diminuição da reserva de glicogênio, o uso de aminoácidos como fornecedores de esqueletos de carbono e aumento da b-oxidação de ácidos graxos. A produção de amônia durante o exercício de alta intensidade é um evento bastante estudado, porém pouco se conhece deste metabolismo em exercício de intensidade moderada. Neste estudo investigamos o metabolismo de amônia durante o exercício de intensidade moderada e longa duração associado à dieta cetogênica como indutores de estresse metabólico. Materiais e Métodos: Os atletas $(n=7)$ foram avaliados segundo diversos parâmetros clínicos e tiveram seu consumo máximo de oxigênio $\left(\mathrm{VO}_{2 m a ́ x}\right)$ e freqüência cardíaca máxima $\left(F C_{\text {máx }}\right)$ individualmente estimados. Os sujeitos permaneceram em dieta cetogênica nas $72 \mathrm{~h}$ prévias ao experimento que aconteceu com intensidade de $60 \%$ da potência desenvolvida no $\mathrm{VO}_{2 \text { máx }}$ e $70 \%-75 \%$ da $\mathrm{FC}_{\text {máx }}$ durante 60 min. Resultados: Houve aumento de $35 \%$ da amonemia basal em resposta a dieta cetogênica. O exercício causou elevação de $250 \%$ na amonemia, coerentemente a uremia basal se elevou $60 \%$ devido à dieta cetogênica, sem mudanças em resposta ao exercício. O urato sérico basal se elevou $10 \%$ sem ser modificado pelo exercício. Não detectamos mudanças na glicemia ou lactatemia durante qualquer fase do estudo. Discussão: Nossos achados parecem indicar que o exercício de intensidade moderada associado à dieta cetogênica pode ser usado como modelo para elevação da amonemia, possibilitando o seu uso como indutor de estresse metabólico para o estudo do metabolismo de amônia.

\section{PALAVRAS-CHAVE}

Hiperamonemia, Lactato, Urato, Sistema Nervoso Central.

\footnotetext{
1 Universidade Federal do Estado do Rio de Janeiro - UNIRIO - Laboratório de Bioquímica de Proteínas - Rio de Janeiro - Brasil

${ }^{2}$ Universidade de Trás-os-Montes e Alto Douro - UTAD - Programa de Pós-Graduação em Educação Física e Desporto - Vila Real - Portugal

${ }^{3}$ Universidade Federal do Rio de Janeiro - UJRJ - Departamento de Biociências e Atividade Física - Laboratório de Biologia Muscular e do Exercício - Rio de Janeiro - Brasil

${ }^{4}$ Universidade Castelo Branco - UCB-RJ - Programa de Pós Graduação em Ciência da Motricidade Humana - Rio de Janeiro - Brasil

${ }^{5}$ Universidade Federal de Uberlândia UFU - Instituto de Genética e Bioquímica - Uberlândia - Brasil
} 


\title{
Moderate-intensity exercise Associated With Ketogenic diet. A mOdel for the Study Of AMMONIA METABOLISM?
}

\begin{abstract}
Introduction: Exercise has been used as a model to study the metabolism of ammonia $\left(\mathrm{NH}_{3}+\mathrm{NH}_{4}^{+}\right)$, which is highly toxic to the central nervous system. $\mathrm{A}$ ketogenic diet leads to several metabolic adaptations to maintain the ATP/ADP ratio, including a lack of glycogen reservoirs, the use of amino acids as carbon skeleton donors and increased $\beta$-oxidation of fatty acids. The formation of ammonia during high-intensity exercise is well studied, but its role in moderateintensity exercise remains unclear. Here we investigate ammonia metabolism during moderate-intensity exercise associated with a ketogenic diet as a model system. Materials and Methods: Athletes $(n=7)$ were physically evaluated and had their maximum oxygen consumption $\left(\mathrm{NO}_{2 \max }\right)$ and heart rate $\left(\mathrm{HR}_{\text {max }}\right)$ determined. The subjects remained on a ketogenic diet $72 \mathrm{~h}$ prior to exercise. They then exercised for $60 \mathrm{~min}$ at a power output of $60 \%$ of that at $\mathrm{VO}_{2 \mathrm{max}}$ and at $70 \%-75 \%$ of $\mathrm{HR}_{\text {max }}$. Results: Basal ammonemia increased by $35 \%$ due to dietetic modifications, while exercise caused a $250 \%$ increase in ammonemia in parallel with this effect. Uremia was increased by $60 \%$ due to the ketogenic diet without response to exercise. We measured a $10 \%$ increase in serum urate that did not change during the exercise protocol. No changes were found in glycemia or lactatemia. Discussion: Our data suggest that moderate-intensity exercise associated with a ketogenic diet can be used to study the increase in ammonemia and as a model to understand ammonia metabolism during metabolic stress.
\end{abstract}

\section{KEYWORDS}

Hiperammonemia, Lactate, Urate, Central Nervous System.

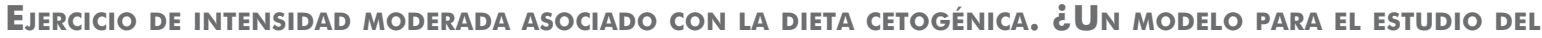 METABOLISMO DE AMONÍACO?}

\section{RESUMEN}

Introducción: El ejercicio tiene sido usado como modelo para el estudio del metabolismo de amonio $\left(\mathrm{NH}_{3}+\mathrm{NH}_{4}^{+}\right)$un metabólico toxico para el sistema nervioso central. La dieta cetogénica lleva a diferentes modificaciones metabólicas para la manutención de la relación ATP/ADP que incluyen el consumo de las reservas de glicógeno, el uso de los esqueletos de carbono de los aminoácidos y el aumento de la b-oxidación de los ácidos grasos. La génesis del amonio durante el ejercicio de alta intensidad es bien estudiado pero poco como acontece durante el ejercicio de intensidad moderada. En este estudio, investigamos el metabolismo de amonio durante el ejercicio de intensidad moderada asociado a una dieta cetogénica como modelo de estudio para el metabolismo de amonio. Materiales y Métodos: Los atletas $(n=7)$ fueran evaluados clínicamente y tuvieran su consumo máximo de oxigeno $\left(\mathrm{NO}_{2 \text { móx }}\right)$ evaluado y su frecuencia cardiaca $\left(\mathrm{HR}_{\text {max }}\right)$ máxima determinada. Los sujetos estuvieran en dieta cetogénica por $72 \mathrm{~h}$ antes del ejercicio que fue hecho a la intensidad de $60 \%$ da potencia desarrollada en el $\mathrm{VO}_{2 \text { máx }}$ y a 70\%-75\% de la HR móx durante 60min. Resultados: La amonemia basal aumento 35\% debido a las modificaciones dietéticas. El ejercicio provoco el aumento de $250 \%$ en la amonemia. Paralelamente, la uremia subió $60 \%$ en respuesta a la dieta cetogénica sin ser modificada por el ejercicio. Medimos un aumento de $10 \%$ en el urato sérico que no fue afectado por el protocolo de ejercicio. No hubo modificaciones en los niveles de glicemia o lactatemia durante todo el protocolo experimental. Discusión: Nuestros datos sugieren que el ejercicio de intensidad moderada asociado a dieta cetogénica puede ser usado para investigar el aumento de la amonemia y puede ser útil como modelo para la comprensión del metabolismo de amonio durante el estrese metabólico.

\section{PALABRAS CLAVE}

Hiperamonemia, Lactato, Urato, Sistema Nervioso Central.

\section{INTRODUÇÃO}

Quando o incremento na contração muscular associada à hidrólise de ATP excede a velocidade de sua ressíntese, há aumento na concentração intracelular de AMP. O AMP é desaminado pela AMPdeaminase produzindo inosina monofosfato (IMP) e amônia (entendida aqui como $\left.\mathrm{NH}_{3}+\mathrm{NH}_{4}^{+}\right)^{1}$. Além disso, o aumento na concentração intracelular de ADP gera aumento no fluxo de entrada de aminoácidos no ciclo do ácido cítrico, através da desaminação oxidativa produzindo amônia e cetoácidos que serão utilizados para a produção de ATP e para a manutenção da glicemia ${ }^{2,3}$.

A dieta cetogênica é caracterizada pela diminuição da ingestão de carboidratos e pode levar a redução das reservas de glicogênio muscular resultando na diminuição do fluxo glicolítico e subsequente produção de lactato ${ }^{4,5,6}$. Consequentemente, a dieta cetogênica aumenta a oxidação de aminoácidos, ácidos graxos e corpos cetônicos para a produção de ATP.

A utilização da dieta cetogênica associada ao exercício aumenta a utilização de aminoácidos como doadores de esqueletos de carbono para formação de ATP ou para a neoglicogênese, aumentando a concentração de amônia livre. No hepatócito, a amônia ativa a hidrólise de glutamato, via glutamato desidrogenase formando 2-oxoglutarato e mais amônia. Este caminho de desaminação oxidativa do glutamato é de vital importância para a interação das vias metabólicas, pois aumenta a 

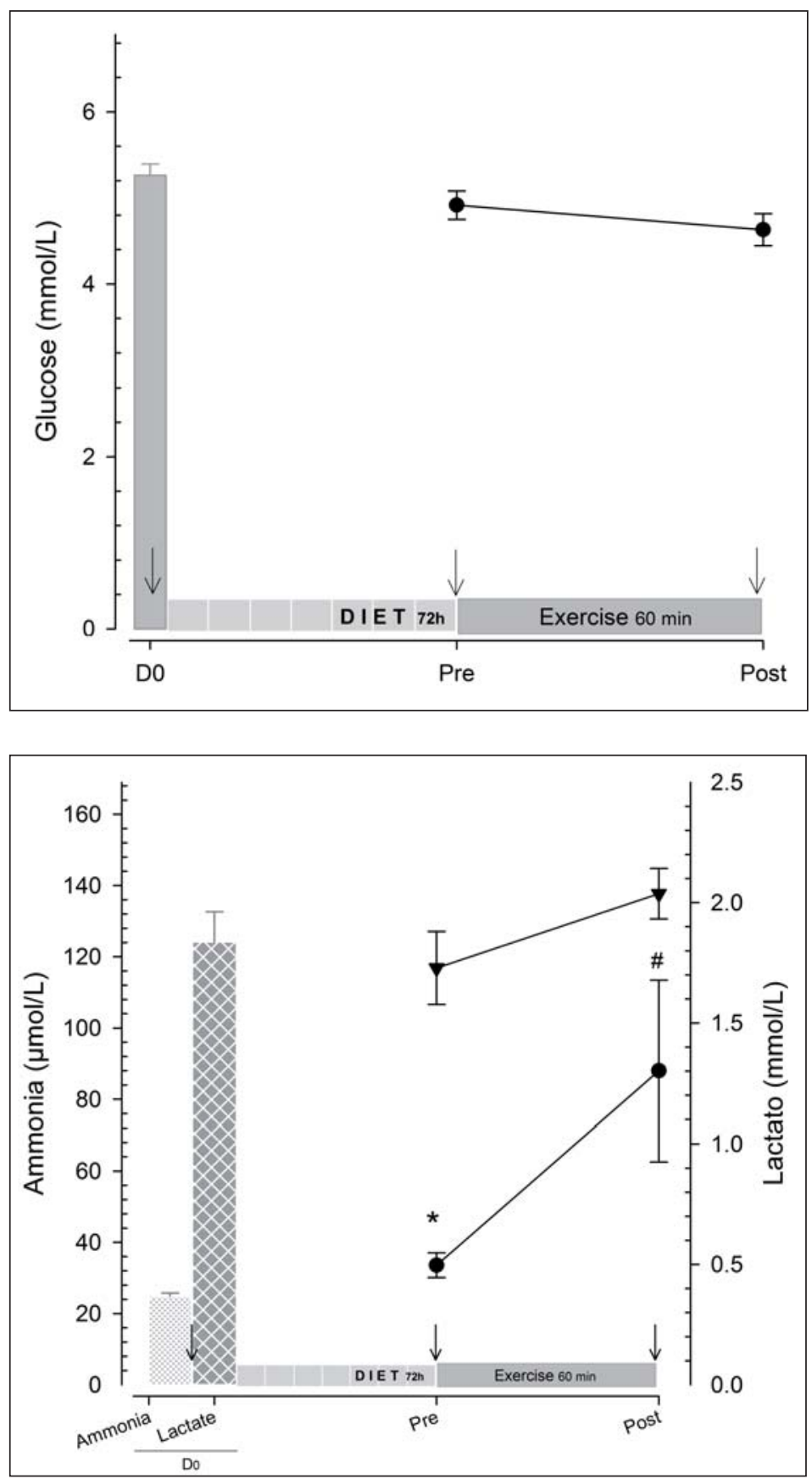

Figura 1 - Resposta glicêmica à dieta e ao exercício. A dieta cetogênica e o exercício não modificaram a glicose sanguínea.

$(\downarrow)$ coleta sanguínea
Figura 2 - A amonemia aumentou em resposta a dieta e ao exercício. A dieta e o exercício agiram sinergicamente na indução da produção de amônia e não modificaram a de lactato.

(.) DO diferente de Pré; (\#) Pré diferente de Pós; (•) amônia; $(\nabla)$ lactato; $(\downarrow)$ ) coleta sanguínea atividade do ciclo do ácido cítrico auxiliando na produção de $A T P^{\mathbf{7}, \mathbf{8}}$. $\bigcirc$ fígado responde rapidamente às mudanças sistêmicas nos níveis de amônia ativando o ciclo da ureia e a síntese de glutamato e glutamina, ${ }^{90}$.

A amônia muscular se difunde para o sangue e pode atravessar livremente a barreira hemato-encefálica ${ }^{11}$ causando alteração na sensibilidade específica ao glutamato e pode justificar a variabilidade na precipitação de fatores e respostas latentes das disfunções mediadas pelo SNC. Tem sido sugerido que níveis elevados de amônia podem estar associados ou ser responsáveis pela fadiga no exercício, falta de coordenação motora, ataxia, torpor que se originam du- rante ou após o exercício ${ }^{12}$. Estes sintomas são semelhantes aos encontrados em diversas doenças hepáticas.

Neste estudo investigamos o metabolismo de amônia usando um modelo de exercício de baixa intensidade associado à dieta cetogênica para aumentar o stress metabólico.

\section{MATERIAIS E MÉTODOS}

\section{Aprovação do estudo}

$\bigcirc$ protocolo experimental atendeu aos requisitos para realização de pesquisa em humanos (Resolução 

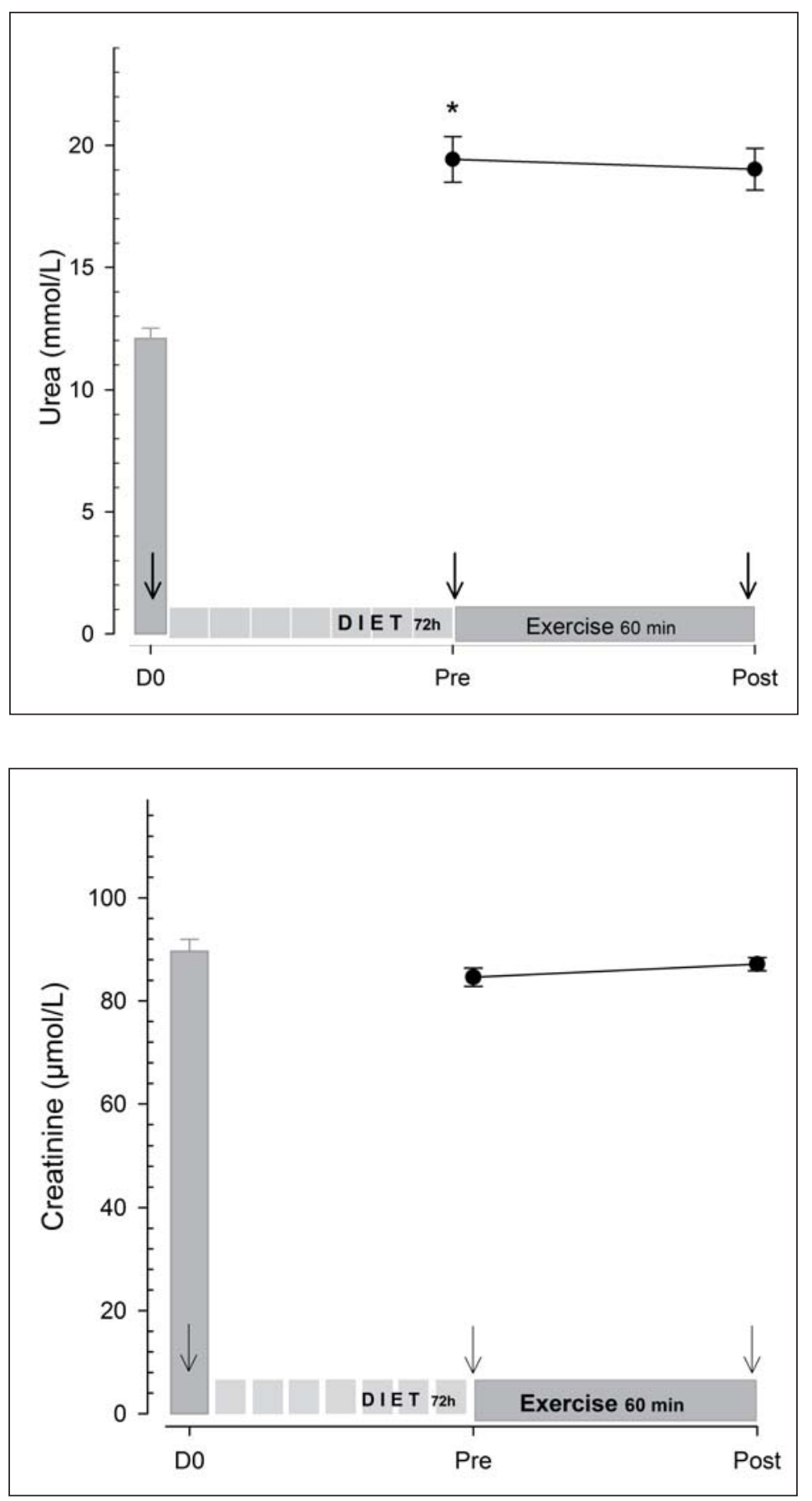

Figura 3 - A ureagênese aumentou em resposta à dieta

(.) DO diferente de Pré; $(\downarrow)$ ) coleta sanguínea
Figura 4 - A creatinina como controle da função renal. As Concentrações de creatinina se mantiveram constantes durante a dieta e pós exercício.

$(\downarrow)$ coleta sanguínea
196/1996 do Conselho Nacional de Saúde, Brasil) que está em conformidade com a declaração de Helsinki (1975, adendo 2000). O estudo foi aprovado pelo Comitê de Ética em Pesquisa da Universidade Federal do Estado do Rio de Janeiro (CEP 1 17/2007).

\section{Amostra}

Participaram desse estudo sete ciclistas profissionais do sexo masculino entre 25 e 35 anos (27,4 43,5 anos). Para assegurar a homogeneidade do grupo foram realizadas avaliações antropométricas, ergoespirométricas
$\left(\mathrm{VO}_{2 \text { máx }}=61,7 \pm 5,9 \mathrm{~mL} \cdot \mathrm{kg}^{-1} \cdot \mathrm{min}^{-1} ; \mathrm{FC}_{\text {máx }}=193 \pm 9 \mathrm{bpm}\right.$; $\left.\mathrm{W}_{\text {máx }}=390 \pm 96 \mathrm{~W}\right)$, clínica e hematológica uma semana antes da sessão exercício (D0). Os ciclistas apresentaram estatura média de 170,0 $0,9 \mathrm{~cm}$; massa corporal média de $64,4 \pm 11,5 \mathrm{~kg}$ e percentual de gordura corporal de $7,5 \pm 2,5 \%$. O grupo não treinou ou fez uso de medicamentos e suplementos alimentares durante o estudo.

\section{Modelo de estudo}

A dieta cetogênica foi iniciada $72 \mathrm{~h}$ antes da sessão de exercício. Os atletas se apresentaram ao laboratório 
pela manha (8h) e, após $12 \mathrm{~h}$ de jejum, foram submetidos coleta sanguínea (Pré).

O exercício foi mantido a $60 \%$ da potência desenvolvida $(234 \pm 58 \mathrm{~W})$ no $\mathrm{VO}_{2 \text { máx' }}$ sendo monitorados individualmente por frequencímetros cardíacos (Polarò) com média de $70 \%$ a $75 \%$ da $\mathrm{FC}_{\text {máx }}$ durante os $60 \mathrm{~min}$ do teste. $O$ estudo foi realizado em temperatura ambiente com média de $25^{\circ} \mathrm{C}$ e umidade relativa do ar média de $62 \%$. A terceira coleta de sangue foi após o teste (Pós).

\section{Dieta cetogênica}

Foi usado o modelo de dieta cetogênica para depleção de glicogênio. $O$ plano de dieta seguiu a restrição da ingestão de carboidratos $72 \mathrm{~h}$ antes do experimento. O curto espaço de tempo serviu para diminuir a chance de mudança na expressão de enzimas em resposta a mudança alimentar. A dieta foi isoenergética, com o mínimo de $1,5 \mathrm{~g} \cdot \mathrm{kg}^{-1}$ (peso atual) de proteínas e continha no máximo de $50 \mathrm{~g}$ de carboidratos por dia. $\bigcirc$ consumo de água foi ad libitum.

\section{Coleta sanguínea}

As amostras de sangue foram coletadas da veia antecubital pré e pós-teste em tubos com EDTA. Imediatamente após a coleta, o sangue foi centrifugado; sendo o plasma separado, congelado e armazenado a $-70^{\circ} \mathrm{C}$, para evitar a perda de material volátil e/ou metabolismo pelos elementos figurados. As coletas e análises sanguíneas foram feitas pelo Laboratório Bittar Ltda (Bittar, Brasil).

Neste artigo, utilizaremos a denominação amônia para referirmos ao somatório das formas $\mathrm{NH}_{3}$ e $\mathrm{NH}_{4}{ }^{+}$.

\section{Estatística}

Os resultados obtidos durante o estudo foram analisados através do Test " $t$ " de Student e o nível de significância considerado foi de $p<0,05$. Os dados estão expressos em média \pm erro padrão.

\section{RESULTADOS}

Para descartar a possibilidade de doenças subclínicas, foi analisado o metabolismo de macronutrientes e hormônios relacionados ao anabolismo. A capacidade do transporte de gases foi verificada através do setor vermelho e da ergoespirometria (Tabela 1). Infecções e lesões teciduais foram descartadas pela análise do setor branco e plaquetas associada aos marcadores enzimáticos clássicos de injúria celular (Tabelas 1 e 2).

Lesões hepáticas, musculares esqueléticas e cardíacas foram avaliadas através da atividade plasmática de enzimas ( $g G T, A S T, A L T, A L P, L D H, C K$ e CKMB) expressas por estes tecidos (Tabela 2). Não houve diferença entre os indivíduos. Todos se encontravam em faixas anteriormente descritas para atletas com nível de treinamento e atividades similares ${ }^{13,14,15}$.

A dieta cetogênica não alterou a glicemia basal dos sujeitos quando comparada com D0, resultado idêntico

Tabela 1 - Análise bioquímica e hematológica dos ciclistas em D0. Os atletas chegaram em jejum de $12 \mathrm{~h}$ para coleta sanguínea. Todos os valores encontrados estão dentro da faixa de normalidade. Os valores estão expressos como média \pm erro padrão.

\begin{tabular}{|c|c|}
\hline Hemograma + Plaquetas & Do \\
\hline Hemácias $\left(x 10^{12} \cdot \mathrm{L}^{-1}\right)$ & $5,2 \pm 0,2$ \\
\hline Hemoglobina (mmol..-1) & $2,3 \pm 0,1$ \\
\hline Hematócrito (\%) & $44,5 \pm 0,9$ \\
\hline VCM (fL) & $85,7 \pm 1,6$ \\
\hline $\mathrm{HCM}(\mathrm{pg})$ & $29,1 \pm 0,7$ \\
\hline $\mathrm{CHCM}\left(\mathrm{g} \cdot \mathrm{L}^{-1}\right)$ & $33,9 \pm 0,2$ \\
\hline RDW (\%) & $13,7 \pm 0,2$ \\
\hline Leucócitos $\left(10^{9} . \mathrm{L}^{-1}\right)$ & $5,1 \pm 4,2$ \\
\hline Basófilos & $0,1 \pm 8,4$ \\
\hline Eosinófilos & $0,2 \pm 0,1$ \\
\hline Linfócitos & $2,1 \pm 0,1$ \\
\hline Monócitos & $0,5 \pm 0,1$ \\
\hline Neutrófilos & $2,8 \pm 0,3$ \\
\hline Plaquetas $\left(\times 10^{9} . \mathrm{L}^{-1}\right)$ & $244,6 \pm 14,9$ \\
\hline Macronutrientes & Do \\
\hline Colesterol Total (mmol.L-1) & $4,5 \pm 0,3$ \\
\hline LDL & $2,6 \pm 0,3$ \\
\hline HDL & $1,3 \pm 0,0$ \\
\hline VLDL & $0,5 \pm 0,1$ \\
\hline Triacilglicerol (mmol..-1) & $1,2 \pm 0,1$ \\
\hline Proteína Total $\left(\mathrm{g} \cdot \mathrm{L}^{-1}\right)$ & $78,3 \pm 1,9$ \\
\hline Albumina $\left(g \cdot \mathrm{L}^{-1}\right)$ & $46,8 \pm 1,3$ \\
\hline Globulina (g. $\left.\mathrm{L}^{-1}\right)$ & $3,4 \pm 1,6$ \\
\hline Relação A/G (g.L-1) & $15,1 \pm 0,9$ \\
\hline Hormônios & DO \\
\hline T3 (mmol. $\left.\mathrm{L}^{-1}\right)$ & $1,7 \pm 0,1$ \\
\hline T3 livre (pmol. $\left.\mathrm{L}^{-1}\right)$ & $6,3 \pm 0,1$ \\
\hline T4 (pmol. $\left.\mathrm{L}^{-1}\right)$ & $95,7 \pm 13,8$ \\
\hline T4 livre (pmol. $\left.\mathrm{L}^{-1}\right)$ & $13,8 \pm 0,6$ \\
\hline TSH (mUI.L-1) & $1,9 \pm 0,3$ \\
\hline $\mathrm{GH}\left(\mu \mathrm{g} \cdot \mathrm{L}^{-1}\right)$ & $0,1 \pm 0,01$ \\
\hline IGF-1 (ng. mL-1) & $183,7 \pm 16,8$ \\
\hline Insulina $\left(\mu \bigcup \mathrm{mL}^{-1)}\right.$ & $5,4 \pm 0,3$ \\
\hline Testosterona (nmol. $\mathrm{L}^{-1}$ ) & $14,5 \pm 0,4$ \\
\hline Testosterona livre (pg. $\mathrm{mL}^{-1}$ ) & $23,2 \pm 1,7$ \\
\hline Leptina (ng. $\mathrm{mL}^{-1}$ ) & $10,5 \pm 1,9$ \\
\hline
\end{tabular}

VCM: volume corpuscular médio; HCM: hemoglobina corpuscular média; CHCM: concentração de hemoglobina corpuscular média; RDW: distribuição do tamanho eritrocitário; LDL: lipoproteínas de baixa densidade; HDL: lipoproteínas de alta densidade; VLDL: lipoproteínas de baixíssima densidade; PCT: procalcitonina; T3: triodotironina; T4: tiroxina; TSH: hormônio estimulante da tireóide; GH: hormônio do crescimento; IGF-1: insulin-like growth factor-1 


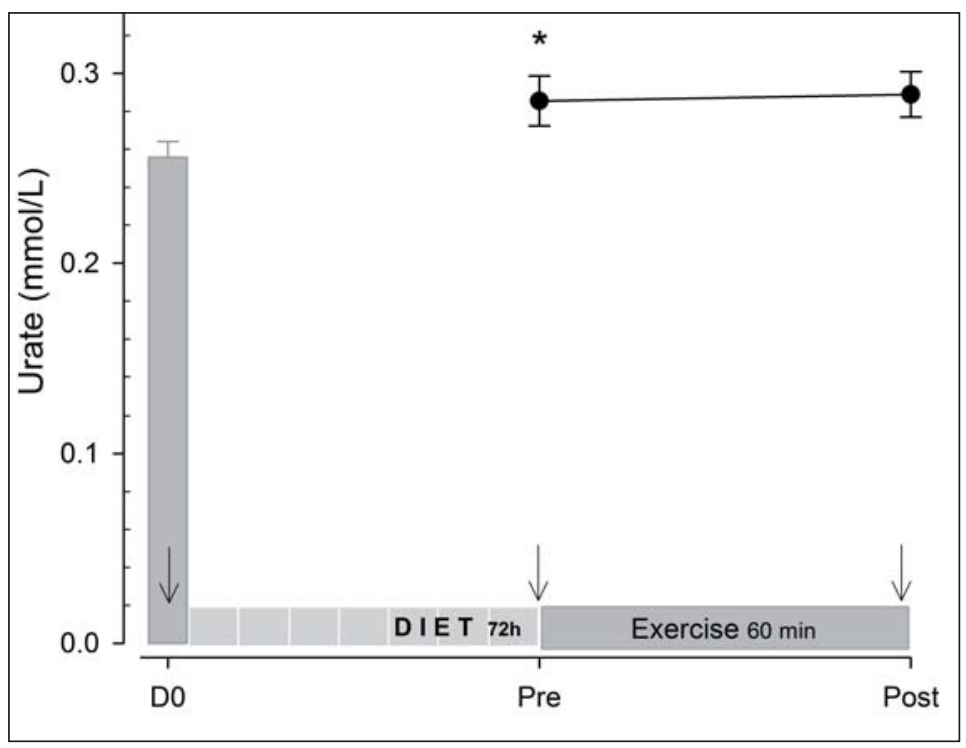

Figura 5 - $O$ urato sérico aumentou em resposta a dieta cetogênica

(.) DO diferente de Pré; $(\downarrow)$ ) coleta sanguínea ao que aconteceu com a lactatemia. $\bigcirc$ exercício não modificou a glicemia ou a lactatemia (Figuras 1 e 2).

A amonemia basal aumentou $35 \%$ em resposta à dieta cetogênica. $\bigcirc$ exercício associado a dieta causou elevação de $250 \%$ na amonemia (Figura 2). Coerente com este achado, a uremia basal aumentou $60 \%$ devido à dieta cetogênica, sem mudanças em resposta ao exercício (Figura 3).

A creatinina sérica não se modificou em resposta a nenhuma das intervenções durante o estudo (Figura 4). Houve aumento de cerca de $10 \%$ do urato sérico basal, sem modificação induzida pelo exercício (Figura 5).

\section{DISCUSSÃo}

A avaliação hematológica e bioquímica prévia foi utilizada para garantir a hemogeneidade metabólica do grupo ${ }^{16}$.

A redução da disponibilidade de glicose provocada pela manipulação dietética induz maior catabolismo de aminoácidos e ácidos graxos para a manutenção da taxa ATP/ADP e continuidade do exercício de longa duração. A dieta cetogênica pode levar a redução das reservas de glicogênio/glicose, do fluxo glicolítico no músculo esquelético ${ }^{4,5,6}$ e induzir a gliconeogênese. A diminuição no fluxo da via glicolítica promove o aumento na concentração de IMP e de intermediários do ciclo do ácido cítrico 17,18,19. Assim, utilizamos, combinados, o incremento de carga e o déficit de glicogênio para aumentar a con-

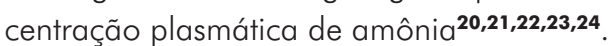

processo anaplerótico aumenta o pool de amônia e esta é exportada para o sangue ${ }^{19}$. No nosso estudo, observamos aumento da amonemia entre DO e o momento pré-exercício pelo maior uso de proteínas na dieta de forma independente do exercício, ratificado pelo aumento associado da uremia.

Medimos 250\% de aumento nas concentrações de amônia em resposta ao exercício associado à dieta. No músculo esquelético em contração intensa, a velocidade aumentada da glicólise eleva a concentração de piruvato que é reduzido a lactato ou Acetil-S-CoA para entrada no Ciclo de Krebs. Ademais, a elevação da síntese concomitante de alanina e glutamina auxilia a manter o fluxo da via glicolítica ${ }^{25}$. Por ser a atividade mitocondrial insuficiente para refosforilar o ADP gerado, há aumento progressivo da gênese de amônia que se difunde para o sangue e pode ser captada por diversos tecidos ${ }^{1}$. No nosso estu-

Tabela 2 - Enzimas marcadoras de injúria hepática e muscular. Não há diferença entre as enzimas nos tempos medidos. Os valores são expressos como média \pm erro padrão.

\begin{tabular}{lccc}
\hline Enzimas (U.L-1) & D0 & Pré & Pós \\
\hline ALT & $25,4 \pm 2,4$ & $24,1 \pm 2,0$ & $25,0 \pm 1,6$ \\
AST & $26,4 \pm 3,8$ & $29,6 \pm 3,0$ & $29,1 \pm 2,5$ \\
$\gamma G T$ & $18,6 \pm 2,6$ & $17,8 \pm 1,8$ & $18,1 \pm 2,0$ \\
FA & $57,0 \pm 3,8$ & $54,4 \pm 3,6$ & $56,1 \pm 3,2$ \\
LDH & $367,6 \pm 41,4$ & $395,0 \pm 44,0$ & $382,8 \pm 34,4$ \\
CK & $295,8 \pm 86,9$ & $283,0 \pm 66,4$ & $291,3 \pm 67,9$ \\
CKMB & $17,7 \pm 3,1$ & $22,6 \pm 3,4$ & $20,3 \pm 2,2$ \\
\hline
\end{tabular}

AST: aspartato aminotransferase; ALT: alanina aminotransferase; $\gamma G T$ : gama glutamiltransferase; FA: fosfatase alcalina; LDH: lactato desidrogenase; CK: creatina quinase total; $C K M B$ : creatina quinase $M B$ 
do, a uremia se elevou em resposta a dieta, no entanto, permaneceu estável durante o exercício, mesmo com subida da amonemia. Este dado é explicável devido ao período estudado, onde não há tempo para metabolismo de amônia e saída da ureia do hepatócito para o sangue.

Não medimos aumento da lactatemia em resposta ao exercício. A gênese de lactato é menor em indivíduos depletados de glicogênio, ocasionando aumento na concentração de ADP. A mudança na relação ATP/ADP ativa a AMP deaminase, aumentando a formação de amônia e o consumo de oxigênio ${ }^{26}$. Tem sido descrito que em eventos de endurance indivíduos com dieta deficiente em carboidratos tem respostas mecânicas inadequadas alcançando rapidamente 0 ponto de fadiga ${ }^{27,28}$.

Lowenstein \& Goodman ${ }^{29}$ demonstraram que a amônia muscular aumenta em até $400 \%$ durante exercício, representando $74 \%$ da produção líquida da amônia, sendo a amônia total formada correspondente à quantidade intramuscular de IMP acumulada, sugerindo que a amônia foi derivada da desaminação de AMP. Durante a atividade física a amônia pode ser produzida a partir da degradação de nucleotídeos com aparecimento concomitante de IMP e urato ${ }^{30}$. O aumento na concentração sérica de urato que é classificado como um evento clássico em situações de jejum prolongado ou diminuição da ingestão de carboidratos. Estudos anteriores em nosso laboratório têm demonstrado que a cinética do aparecimento da ureia e urato no sangue obedecem a uma cinética mais tardia do que a da amônia (Bessa et al., manuscrito em preparação). Nossos achados podem indicar que o aumento do urato sérico antes do exercício pode ser causado pelo aumento da ingestão de alimentos ricos em bases púricas a principal fonte para a formação de IMP e urato ${ }^{31}$.

Nossos dados parecem indicar que o exercício de intensidade moderada associado a dieta cetogênica pode ser usado como modelo para elevação da amonemia, possibilitando o seu uso como indutor de estresse metabólico para o estudo do metabolismo de amônia.

\section{REFERÊNCIAS}

1. Graham TE, MacLean DA. Ammonia and amino acid metabolism in human skeletal muscle during exercise. Am J Physiol Pharmacol. 1990;70:132-41.

2. Graham TE, Turcotte LP, Kiens B, Richter EA. Effect of endurance training on ammonia and amino acid metabolism in humans. Med Sci Sports Exerc. 1997;29(5):646-53.

3. Rossi L, Tirapegui J. Aspectos atuais sobre exercício físico, fadiga e nutrição. Paul Educ Fís. 1999;13(1):67-82.

4. Gerich JE. Control of glycaemia. Baillieres Clin Endocrinol Metab. 1993;7(3):551-86.

5. Spriet LL, Howlett RA, Heigenhauser GJ. An enzymatic approach to lactate production in human skeletal muscle during exercise. Med Sci Sports Exerc. 2000;32(4):756-63.

6. Newsholme P, Lima MM, Procopio J, et al. Glutamine and glutamate as vital metabolites. Braz J Med Biol Res. 2003;36(2):153-63.
7. Jones EA. Ammonia, the GABA neurotransmitter system, and hepatic encephalopathy. Metabolic Brain Disease. 2002;17(4):275-81.

8. Monfort P, Kosenko E, Erceg S, Canales JJ, Felipo V. Molecular mechanism of acute ammonia toxicity: role of NMDA receptors. Neurochem Int. 2002;41:95-102

9. Buttrose MD, McKellar D, Welbourne TC. Gut-liver interaction in glutamine homeostasis: portal ammonia role in uptake and metabolism. Am J Physiol. 1987;252(6 Pt 1):E746-50.

10. Damink WMO, Detz NEP, Dejong CHC, Soeters PB, Jalan R. Interorgan ammonia metabolism in liver failure. Neurochem Int. 2002;41:177-88.

11. Guezennec CY, Abdelmalki A, Serrurier-Merino D, Bigard X, Berthelot BM, Pierard C, et al. Effects of prolonged exercise on brain ammonia and acids. Int J Sports Med. 1998;19:323-7.

12. Banister EW, Cameron BJ. Exercise-induced hyperammonemia: Peripheral and central effects. Int J Sports Med. 1990;11(2):S129-142.

13. Bassini-Cameron A, Sweet E, Bottino A, Bittar C, Veiga C, Cameron LC. Effect of caffeine supplementation on haematological and biochemical variables in elite soccer players under physical stress conditions. $\mathrm{Br} J \mathrm{Sports}$ Med. 2007;41(8):523-30; discussion 530. Epub 2007 May 1.

14. Bessa A, Nissenbaum M, Monteiro A, Gandra PG, Nunes LS, BassiniCameron A, Werneck-de-Castro JP, de Macedo DV, Cameron LC. Highintensity ultraendurance promotes early release of muscle injury markers. Br J Sports Med. 2008;42(11):589-93. Epub 2008 Jan 18.

15. Lazarim FL, Antunes-Neto JM, da Silva FO, Nunes LA, Bassini-Cameron A, Cameron LC, et al. The upper values of plasma creatine kinase of professional soccer players during the Brazilian National Championship. J Sci Med Sport. 2009;12(1):85-90. Epub 2008 Feb.

16. Bassini-Cameron A, Monteiro A, Gomes A, Werneck-de-Castro JP, Cameron L. Glutamine protects against increases in blood ammonia in football players in an exercise intensity-dependent way. Br J Sports Med. 2008;42(4):260-6.

17. Spencer MK, Katz A. Role of glycogen in control of glycolisis and IMP formation in human muscle during exercise. Am J Physiol. 1991;260 (Endocrinol Metab 23):E859-E864

18. Roeykens J, Magnus RR, Meeusen K, Meirleir D. Bood ammonia - Heart rate relationship during graded exercise is not influenced by glycogen depletion. Int J Sports Med. 1998; 19:26-31

19. Gibala MJ; Peirce N, Constantin-Teodosiu D, Greenhaff F. Exercise with low muscle glycogen augments TCA cycle anaplerosis but impairs oxidative energy provision in humans. J Physiol. 2002;540(3):1079-86.

20. Hankard RG, Haymond MW, Darmaun D. Role of glutamine as a glucose precursor in fasting humans. Diabetes. 1997;46(10):1535-41.

21. Chen $P$, Harcum SW. Effects of amino acid additions on ammonium stressed CHO cells. J Biotechnol. 2005;117(3):277-86.

22. Modre-Osprian R, Osprian I, Tilg B, Schreier G, Weinbergerkm, Graber A. Dynamic simulations on the mitochondrial fatty acid beta-oxidation network. BMC Syst Biol. 2009;3:2

23. Felig P, Marliss EB, Cahill Jr GF. Metabolic response to human growth hormone during prolonged starvation. J Clin Invest. 1971;50(2):411-21.

24. Smith DJ, Norris SR. Changes in glutamine and glutamate concentrations for tracking training tolerance. Med Sci Sports Exerc. 2000;32(3):684-9.

25. Thomas C, Perrey S, Lambert K, Hugon G, Mornet D, Mercier J. Monocarboxylate transporters, blood lactate removal after supramaximal exercise, and fatigue indexes in humans. J Appl Physiol. 2005;98(3):804-9

26. Schulz $H$, Heck $H$. Glicogen depletion as indication for determination in exercise testing. Europ. J. of Sport Scien. 2003;3(3):1-9.

27. Chicharro JL, Vaquero AF, Tello R, Pérs M, Lúcia A. Relationship between lactate and ammonia thresholds in Heart transplant patients. Chest. 1996; 110:693-7.

28. Ament W, Huizenga JR, Mook GA, Gips CH, Verkerke GJ. Lactate and ammonia concentration in blood and sweat during incremental cycle ergometer exercise. Int J Sports Med. 1997;18(1):35-9.

29. Lowenstein JM, Goodman MN. The purine nucleotide cycle in skeletal muscle Fed Proc. 1978;37(9):2308-12.

30. Thompson WJ, Appleman MM. Characterization of cyclic nucleotide phosphodiesterases of rat tissues. The J of Biol Chemistry. 1971;246(10):3145-50.

31. Brule D, Sarwar G, Savoie L, Campbell J, Van Zeggelaar M. Differences in uricogenic effects of dietary purine bases, nucleosides and nucleotides in rats. J Nutr. 1988;118(6):780-6. 